\title{
Comparison of Left Ventricular Curvedness Derived from CMR Imaging with the Wall Motion Score Index for Male Patients after First-Time Myocardial Infarction
}

\author{
Soo-Kng Teo ${ }^{1 *}$, Xiaodan $\mathrm{Zhao}^{2}$, Ru-San Tan ${ }^{2,3}$, Liang Zhong ${ }^{2,3}$, Yi Su ${ }^{1}$ \\ ${ }^{1}$ Institute of High Performance Computing, A*STAR, Singapore \\ ${ }^{2}$ National Heart Centre, Singapore \\ ${ }^{3}$ Duke-NUS Graduate Medical School, Singapore
}

\begin{abstract}
The wall motion score index (WMSI) is an important clinical measure to assess the aggregate function of left ventricle $(L V)$ wall segments after myocardial infarction (MI). Compared to global LV ejection fraction, WMSI provides additional information about regional functions that corresponds to myocardium contractility. Studies have shown that the WMSI yields powerful prognostic information after MI. However, one limitation of the WMSI is that manual assessment has to be performed by clinicians resulting in potential intra- and inter-observer variabilities.

In this study, we compared the geometry-derived curvedness at end-systole based on cardiac magnetic resonance (CMR) imaging with clinical WMSI in a group of 25 male patients presenting with first-time MI. Our computational method for calculating curvedness has the following advantages: it is automated and robust for a given set of inputs.

Comparing across basal, mid and distal segments, the mean values of curvedness at end-systole for segments with $W M S I=1$ (normokinetic) were significantly different compared to segments with WMSI = 3 (akinetic) and above (p-value <0.05, 1-way ANOVA). We also observed significant difference in curvedness at endsystole for segments with WMSI $=1$ compared to segments with WMSI $=2$ (hypokinetic) at the mid segments (p-value <0.05, 1-way ANOVA). Our results suggest that automatically-generated curvedness may potentially be used for correlating to manually-assessed WMSI for patients after MI. Future work will include expanding the sample size of the patient group to validate our initial results.
\end{abstract}

\section{Introduction}

The wall motion score index (WMSI) is an important clinical measure to assess the aggregate function of left ventricle (LV) wall segments after myocardial infarction (MI). Such aggregate functional assessment is important for patient stratification and subsequent treatment strategy, follow-up and disease prognosis (such as the 1year mortality risk) [1]. Compared to global LV ejection fraction (LVEF) which only provides a global assessment of LV function, WMSI provides additional insights into regional function corresponding to the active myocardium contractility.

Clinical assessment of the WMSI is routinely performed using 2D or 3D echocardiography and characterized using the standard 16-segment American Heart Association (AHA) nomenclature [2]. Each individual LV segment is assigned a semi-quantitative score to reflect its systolic function based on the visual inspection of the myocardium motion and its thickening (as a proxy for contractility) during systole. This score is defined on a 5-point scale based on the following criteria: score $1=$ normokinesis or hyperkinesis; score $2=$ hypokinesis; score 3 = akinesis; score $4=$ dyskinesis and score $5=$ aneurysm. The mean WMSI is then calculated by taking the average of the individual segment scores, thereby providing a proxy for the aggregate LV function. Previous studies have demonstrated that this mean WMSI have the potential to be used in a wide variety of clinical applications such as (i) independent predictor of death and hospitalization after acute MI [3], (ii) prediction of global LV ejection fraction [4] and (iii) predicting final infarct size in patients with non-ST-segment-elevation MI [5].

Despite the ease of WMSI computation using echocardiography and its potential applications, there are several inherent limitations to this approach of assessing LV function. The first limitation pertains to the use of echocardiography as an imaging modality for assessing myocardium motion and thickening. The American Society of Echocardiography and the European Association of Cardiovascular Imaging in their recommendation states that myocardium motion in 
individual segments can potentially be affected by tethering to adjacent segments and the overall motion of the LV. Hence the assessment of individual segment score should utilize multiple views and focus on the regional thickening to differentiate passive deformation from active deformation caused by the contractility of the myocardium [6]. The second limitation pertains to the reproducibility of results obtained using echocardiography, which is dependent on the skills of the operator and the quality of the acoustic window acquired during imaging [7]. As such, computation of WMSI using other imaging modality such as cardiac magnetic resonance (CMR) and multidetector CT imaging are gaining traction. However, regardless of the imaging modality used for acquiring cardiac motion, one outstanding limitation of the WMSI is that manual assessment performed by clinicians is still required. This method of assessment can still result in potential intraand inter-observer variabilities especially among multicenter trials involving large number of patients and reporting clinicians.

In this study, we proposed the use of a 3D geometryderived index for assessing the regional function of the $\mathrm{LV}$ that is the curvedness index at end-systole $\left(\mathrm{C}_{\mathrm{ES}}\right)$. Our computational method for calculating curvedness has the following advantages: it is automated and robust for a given set of inputs. Our hypothesis is that $\mathrm{C}_{\mathrm{ES}}$ can be used to assess the regional $\mathrm{LV}$ functions in a similar manner as the WMSI. To test this hypothesis, we compared $\mathrm{C}_{\mathrm{ES}}$ computed based on CMR imaging with clinical WMSI in a group of 25 male patients presenting with first-time MI. The objective is to demonstrate that the values of $\mathrm{C}_{\mathrm{ES}}$ is correlated to the WMSI score for the individual segments. In addition, we also compare the correlation of both the mean WMSI and mean $C_{E S}$ across all 16 segments in the $\mathrm{LV}$ to the global LVEF to investigate if the aggregation of regional functions can indeed be used to predict global function.

\section{Methods}

The group of MI patient for this study belongs to a sub-group of MI patients recruited as part of their treatment protocol at the National Heart Centre Singapore. The population characteristics of the entire patient group and the imaging protocol has already been described in our previous work [8]. This sub-group of MI patients were selected for this study because of the availability of the WMSI.

In each subject, regional curvedness analysis was performed on reconstructed $3 \mathrm{D}$ geometry of the LV endocardial surface derived from contouring of CMR images for the entire cardiac cycle, and regional functions characterized using standard 16-segment AHA nomenclature. Further elaboration of the reconstruction methodology and the computational approach for calculating curvedness (based on an analytic approach using a local surface patch-fitting method) can be found in our previous works $[9,10]$. The robustness and reproducibility of our approach has also been demonstrated in our previous work [8]. All patients gave informed consent and were recruited without consideration of gender or ethnicity. The protocol used in this study was approved by the SingHealth Centralised Institutional Review Board.

\section{Results and Discussions}

The regional curvedness for all $16 \mathrm{LV}$ segments were computed from the 3D reconstructed geometry. For analysis, we correlate the $\mathrm{C}_{\mathrm{ES}}$ against the WMSI of the individual segments aggregated across the basal (segments 1 to 6), mid (segments 7 to 12) and distal (segments 13 to 16) regions. This stratification by segment position was required as the curvedness values at the distal segments are higher as compared to basal and mid segments; arising as a result of the tapered shape of the LV with the distal segments resulting in higher curvature at the distal region. The results are visualized using box-whisker plot in Figure 1. Comparing across basal, mid and distal segments, $\mathrm{C}_{\mathrm{ES}}$ for segments with WMSI $=1$ (normokinetic) were significantly different compared to segments with WMSI $=3$ (akinetic) and above ( $p$-value $<0.05$, 1-way ANOVA). We also observed significant difference in $\mathrm{C}_{\mathrm{ES}}$ for segments with $\mathrm{WMSI}=1$ compared to segments with WMSI $=2$ (hypokinetic) at the mid segments ( $p$-value <0.05, 1-way ANOVA). Generally, the values of $\mathrm{C}_{\mathrm{ES}}$ decreased with increasing WMSI as indicated by the decreasing median $\mathrm{C}_{\mathrm{ES}}$ in Figure 1. This trend is observed across all regions (basal, mid and distal). Our results suggest that automatically-generated $\mathrm{C}_{\mathrm{ES}}$ may potentially be used for assessing regional myocardium contractility and systole function for patients after MI, providing an alternative to the WMSI.

We also correlate the mean WMSI and mean $\mathrm{C}_{\mathrm{ES}}$ across all 16 segments in the LV to the global LVEF for this group of patients. The results are shown in the scatter plots in Figure 2. From the figure, it can be seen that $\mathrm{C}_{\mathrm{ES}}$ has a better correlation with the global LVEF. Using a linear regression fit, the $R^{2}$ for $\mathrm{C}_{\mathrm{ES}}$ is 0.79 whereas the $R^{2}$ for mean WMSI is 0.65 , suggesting that the mean $\mathrm{C}_{\mathrm{ES}}$ has higher sensitivity to the global LV function. We hypothesize that this is a result of the WMSI being a semi-quantitative score (the scoring is discrete) as compared to $\mathrm{C}_{\mathrm{ES}}$ being a continuous quantitative score.

\section{Clinical Applicability}

One possible clinical application for using the $\mathrm{C}_{\mathrm{ES}}$ is for the assessment of myocardium contractility in all 16 
segments during end-systole. This will allow the clinician to determine the location of the damage to the myocardium arising from the MI without usage of any contrast agent (e.g., late gadolinium-enhanced CMR for visualizing the degree and location of the infarction) or radionuclide tracer. Our approach has the potential to offer clinicians with new insights into the regional systole function and myocardium contractility after an infarction.
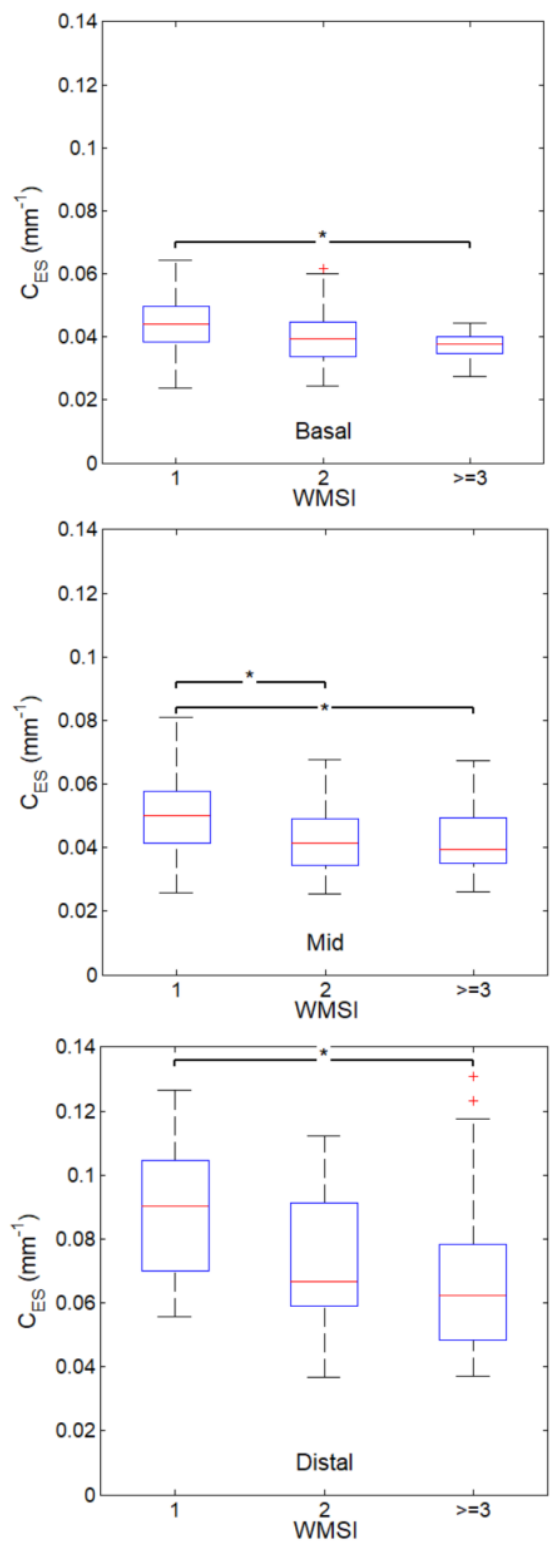

Figure 1. Box-whisker correlating the WMSI with $\mathrm{C}_{\mathrm{ES}}$ for the basal region (top), mid-cavity region (middle) and distal region (bottom). In each box, the central mark (in red) is the median, the edges of the box are the 25th and 75th percentiles and the whiskers extend to approximately +2.7 standard deviation coverage if the data are normally distributed. Refer to main text for discussion of the results $(* p<0.05)$.
Such insights will be essential for both diagnosis (in terms of disease stratification) and patient management.
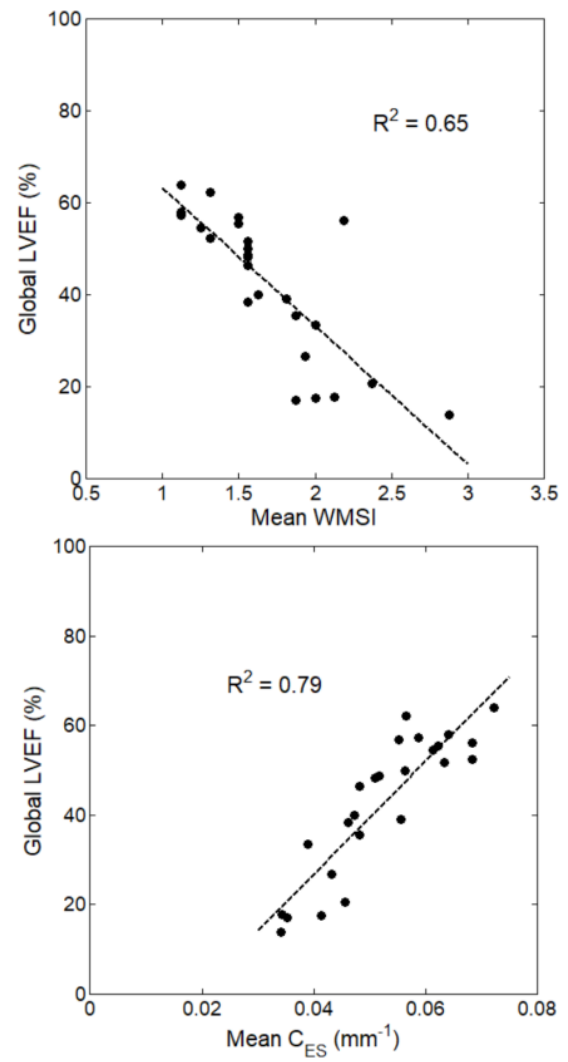

Figure 2. Correlation between the mean WMSI (top) and mean $\mathrm{C}_{\mathrm{ES}}$ (bottom) to the global LVEF. The $\mathrm{C}_{\mathrm{ES}}$ has a better correlation with the global LVEF. A linear functional is used to fit the data.

\section{Limitations}

Our approach for computing the regional curvedness is dependent on the segmentation of the endocardial surface from the CMR images. Currently, this segmentation is performed manually and can be potentially timeconsuming. In addition, there is also a degree of variabilities in the contouring of the endocardial surface across observers though our previous work had demonstrated that our approach is robust to variation in the segmented input contours provided by the user [8]. The development of automated image segmentation techniques utilizing shape-based interpolation, superresolution and deep-learning algorithms can potentially be used to automate the segmentation of the LV endocardial surface and to reduce the variability of the segmented contours (through reducing user inputs).

Also, we acknowledge that the statistical power of this study is limited due to the number of patients recruited (25 patients). Hence, the results reported will still need to be validated on a larger cohort of patients including 
female patients to investigate if there is any gender differences in the $\mathrm{C}_{\mathrm{ES}}$. It will also be interesting to investigate if $\mathrm{C}_{\mathrm{ES}}$ can be used to predict functional recovery in patients after the initial MI (such as correlating the final infarct size in patient after 1 year to the $\mathrm{C}_{\mathrm{ES}}$ computed immediately post the initial MI). Such longitudinal studies can potentially impact the clinical management and treatment of patients with acute MI by stratifying patients based on the recovery prospect.

\section{Conclusions}

We have established a computational approach to compute the regional curvedness values at end-systole $\left(\mathrm{C}_{\mathrm{ES}}\right)$ using the standard 16-segment AHA nomenclature by reconstructing the $3 \mathrm{D}$ geometries of the $\mathrm{LV}$ throughout the entire cardiac cycle. From this study, we observed the following results: (i) $\mathrm{C}_{\mathrm{ES}}$ for segments with WMSI $=1$ were significantly different compared to segments with WMSI $=3$ (akinetic) and above and (ii) the values of $\mathrm{C}_{\mathrm{ES}}$ decreased with increasing WMSI.

\section{Acknowledgements}

This work was supported in part by research grants from the Agency for Science, Technology and Research (A*STAR), SERC (Science and Engineering Research Council) Biomedical Engineering Programme grant, 092 1480071 and 1321480012.

\section{References}

[1] Gerber TC, Nehrenbeck T, Allison T, Mullan BP, Rumberger JA, Gibbons RJ. Comparison of measurement of left ventricular ejection fraction by Tc-99m sestamibi first-pass angiography with electron beam computed tomography in patients with anterior wall acute myocardial infarction. Am J Cardiol 1999; 83:1022-6.

[2] Cerqueira MD, Weissman NJ, Dilsizian V, Jacobs AK, Kaul S, Laskey WK, Pennell DJ, Rumberger JA, Ryan T, Verani MS; American Heart Association Writing Group on Myocardial Segmentation and Registration for Cardiac Imaging. Standardized myocardial segmentation and nomenclature for tomographic imaging of the heart. A statement for healthcare professionals from the Cardiac Imaging Committee of the Council on Clinical Cardiology of the American Heart Association. Circulation. 2002; 105(4):539-42.

[3] Møller JE, Hillis GS, Oh JK, Reeder GS, Gersh BJ, Pellikka PA. Wall motion score index and ejection fraction for risk stratification after acute myocardial infarction. Am Heart J. 2006; 151(2):419-25.

[4] Palmieri V, Russo C, Buonomo A, Palmieri EA, Celentano A. Novel wall motion score-based method for estimating global left ventricular ejection fraction: validation by realtime 3D echocardiography and global longitudinal strain. Eur J Echocardiogr. 2010; 11(2):125-30.

[5] Eek C, Grenne B, Brunvand H, Aakhus S, Endresen K, Hol
PK, Smith HJ, Smiseth OA, Edvardsen T, Skulstad H. Strain echocardiography and wall motion score index predicts final infarct size in patients with non-ST-segmentelevation myocardial infarction. Circ Cardiovasc Imaging. 2010; 3(2):187-94.

[6] Lang RM, Badano LP, Mor-Avi V, Afilalo J, Armstrong A, Ernande L, Flachskampf FA, Foster E, Goldstein SA, Kuznetsova T, Lancellotti P, Muraru D, Picard MH, Rietzschel ER, Rudski L, Spencer KT, Tsang W, Voigt JU. Recommendations for cardiac chamber quantification by echocardiography in adults: an update from the American Society of Echocardiography and the European Association of Cardiovascular Imaging. J Am Soc Echocardiogr. 2015; 28(1):1-39.e14.

[7] Malm S, Frigstad S, Sagberg E, Larsson H, Skjaerpe T. Accurate and reproducible measurement of left ventricular volume and ejection fraction by contrast echocardiography: a comparison with magnetic resonance imaging. J Am Coll Cardiol 2004; 44:1030-5.

[8] Teo SK, Vos FJA, Tan RS, Zhong L, Su Y (2015) Regional ejection fraction and regional area strain for left ventricular function assessment in male patients after first-time myocardial infarction. J R Soc Interface 12:20150006.

[9] Zhong L, Su Y, Yeo SY, Tan RS, Ghista DN, Kassab G. Left ventricular regional wall curvedness and wall stress in patients with ischemic dilated cardiomyopathy. Am. J. Physiol. Heart Circ. Physiol. 2009: 296, H573-H584.

[10] Su Y, Zhong L, Lim CW, Ghista D, Chua T, Tan RS. A geometrical approach for evaluating left ventricular remodeling in myocardial infarct patients. Comput Methods Programs Biomed. 2012; 108(2):500-10.

Address for correspondence.

Soo-Kng Teo.

1 Fusionopolis Way, \#16-16 Connexis, Singapore 138632

teosk@ihpc.a-star.edu.sg 